Granular Matter manuscript No.

(will be inserted by the editor)

\title{
Stress response inside perturbed particle assemblies
}

\author{
Leonardo E. Silbert
}

Submitted;

\begin{abstract}
The effect of structural disorder on the stress response inside three dimensional particle assemblies is studied using computer simulations of frictionless sphere packings. Upon applying a localised, perturbative force within ' the packings, the resulting Green's function response is mapped inside the different assemblies, thus providing an explicit view as to how the imposed perturbation is transmitted through the packing. In weakly disordered arrays, the resulting transmission of forces is of the double-peak variety, but with peak widths scaling linearly with distance from the source of the perturbation. This behaviour is consistent with an anisotropic elasticity response profile. Increasing the disorder distorts the response function until a single-peak response is obtained for fully disordered packings consistent with an isotropic description.
\end{abstract}

Keywords Force Perturbations - Stress Response

PACS 61.43.-j $\cdot$ 81.70.Bt $\cdot$ 83.80.Fg

\section{Introduction}

The internal stresses of a particulate medium, such as a sandpile, determine mechanical stability and structural robustness. How these stresses are distributed can have a profound effect on the way such a system responds to external loading. This is a challenging problem which, one hopes, will lead to a better understanding of the transition between quiescence and catastrophic failure, such as a collapsing grain silo or the onset of an avalanche.

Over the past decade or so, it has come to light that the way forces are transmitted through a disordered pile of grains occurs in a rather inhomogeneous manner [23]. It

Department of Physics, Southern Illinois University, Carbondale, IL 62901, USA,

E-mail: 1silbert@physics.siu.edu seems that it is very much the particulate nature of a granular material that lends itself to the emergence of strongly heterogeneous force networks in disordered packings. Consequently the nature of stress transmission in response to external loading might also exhibit unusual properties due to granularity. These issues have led to renewed interest in describing how stresses are transmitted through particulate matter.

Whereas classical, isotropic, elasticity theory [26; 24] accurately describes the stress state of continuous media, when the discrete nature of the constituent particles influence the way a system responds, it is unclear how one should proceed. So much so that recent simulations of LennardJones glasses have shown how the discrete nature of the amorphous glass influences the elastic properties of the material [43; 39; 27; 28]. Thus, in the context of jammed particulate media, the humble sandpile has thus come to represent the paradigm of an unusual state of matter exhibiting heterogeneous force distributions.

One of the simplest methods to access information about the stress state of a material is to measure the Green's function response to a localised perturbation. For a granular packing, this would involve applying a small force to the central particle at the top of the packing then measuring the pressure at the bottom of the packing in response to this localised external perturbation. To remain in the linear regime, the applied force perturbation must be sufficiently small so as to avoid rearrangement of the grains from their original positions. This is the approach taken in several experimental and simulation studies to date.

Some of the more striking studies involved the direct visualisation of the force networks in two dimensional (2D) packings using photoelastic particles [14; 15; 36], and their micro-displacements [7; 29]. Direct imaging and the subsequent analyses allows one to determine how the contact and/or force network responds when a point force is ap- 
plied to the packing. Presently, more indirect methods are required for inferring the way forces are transmitted through $3 D$ packings $[33 ; 30 ; 38 ; 44$ ], simply due to the fact that granular packs are inconveniently opaque. However, there is a clear distinction between the response properties of disordered grain piles [33] and ordered arrays [30]. There are also numerous analytical and numerical works $111 ; 12 ; 10$; 25; 8 ; $37 ; 22 ; 21 ; 18 ; 13 ; 6 ;$ 31; 5; 19; 16; 42], predicting how forces are transmitted in response to such perturbations.

As a précis to the above, also see Ref. [20], it is now appreciated that granular materials have the uncanny ability to respond in a variety of ways. Isotropic, single-peak response profiles mimic the manner in which an elastic medium behaves as described by partial differential equations of the elliptic class. The isotropic elastic stress state, $\sigma$, of a material in response to a localised load, $F$, on an infinite half space is described by the Boussinesq equation [24],

$\sigma=\frac{3 F}{2 \pi} \frac{z^{3}}{\left(z^{2}+r^{2}\right)^{5 / 2}}$

where $z$ is the vertical distance, or depth, from the source of the force perturbation, and $r$ is the planar distance from the axis of $F[26 ; 24]$. Most of the experiments and simulations performed to date are constructed in such a manner as to directly test the validity of this theory and its $2 D$ analogue the Flamant equation. Under some conditions, strongly anisotropic, double-peak response profiles have been reported. These types of response profiles can be indicative of anisotropic, elastic behaviour, and therefore formally belong to an elliptic description. In the extreme case, double-peak behaviour is representative of hyperbolic, wave-like propagative models, whereby the stresses propagate along characteristic pathways analogous to light rays. In between, is a diffusive parabolic model that seems to be losing favour despite playing a seminal role in describing force heterogeneities in granular media. Moreover, a crossover between different response functions can occur, even within the same pile, depending on structure, particle friction coefficient, distance from the source of the perturbation, and the magnitude of the imposed force. Typically, however, ordered grain configurations result in anisotropic response features, whereas disordered systems usually conform to an isotropic picture.

As far as $2 D$ results go, many of the features described above can be accommodated within the framework of $2 D$ anisotropic elasticity theory [17; 32]. In fact, a parameter space of anisotropic elasticity theory was proposed [32] that allows for the appearance of either single-peak or doublepeak response functions within the same formalism. Simply put, anisotropy in the force/contact network promotes an anisotropic response. One of the key concerns, therefore, is the ability to distinguish between the elastic and wave-like response profiles.
Clearly there are many factors that influence the nature of the resulting response. It would seem prudent to investigate the underlying factors that have the greatest influence in determining the response properties. This is precisely the approach taken in this study. Structure, or the arrangement of the particles that constitute the packing, plays a dominant role. The results presented here are the first to investigate the manner in which the stresses inside $3 D$ particle packings are transmitted in response to localised force perturbations, and how varying the disorder affects the response.

\section{Simulation Model}

The computer experiments reported here are designed to systematically study how structure influences stress transmission in response to localised perturbations. To that end, the simulations are carried out on a model system: three dimensional, monodisperse, non-cohesive, frictionless, soft-sphere packings, with fully periodic boundary conditions, in the absence of gravity. Generation of the initial packings has been described in detail elsewhere [34; 35]. To summarise, $N$ spheres of diameter $d$ and mass $m$, were arranged into a face-centred cubic (fcc) array. The packing fraction, $\phi$, of the particle assemblies was fixed at $\phi=0.742$, just above that of a hard-sphere fcc array, $\phi_{\mathrm{fcc}}=\sqrt{2} \pi / 6$. Two particles, $i$ and $j$, are defined to be contact neighbours and interact through a short-range, purely repulsive, force when $r_{i j}<d$, where $r_{i j}$ is their centre-centre separation. The particles interact via a Hookean force law, $f_{i j}=k\left(d-r_{i j}\right)$, for $r_{i j}<d$, and zero otherwise, i.e. a one-sided linear spring. In this study, the particle stiffness $k$ merely parameterises the force scale and for convenience is set to unity, along with $d$ and $m$. Distances are reported in units of $d$ and all other quantities are appropriately non-dimensionalised.

Configurations with different amounts of disorder were generated by adding defects to the original fcc array. In this study, these defects were introduced by randomly removing particles from the original array then allowing the configuration to relax into a different mechanically stable state at the same $\phi$. Removing more particles prior to the relaxation process resulted in more disorder. As a result the number of particles varied from $N=16384$ for the FCC lattice down to $N=10384$ for the most random packings studied. Consequently, the size of the simulation cube, $L$, ranged from $19.4 d<L<22.6 d$ The amount of disorder was quantified by the coordination number $z$ (average number of contacting neighbours), given in Table 1, which varied from 12 for the fcc array, to below 9 for the most disordered packing. Other measures of the packings corroborated the gradual transition from ordered to disordered configurations [34;35]. This configuration-generation protocol provided a suitable manner in which to control the amount of disorder. The pack- 
Table 1 Configurations are labelled by $C i$ and their coordination number $z$. The fcc array has a value $=12$.

\begin{tabular}{c|ccccccc}
\hline$C i$ & $F C C$ & $\mathrm{C} 2$ & $\mathrm{C} 4$ & $\mathrm{C} 6$ & $\mathrm{C} 8$ & $\mathrm{C} 10$ & $\mathrm{C} 12$ \\
\hline$z$ & 12 & 11.85 & 11.71 & 11.22 & 10.68 & 10.29 & 8.92 \\
\hline
\end{tabular}

ings are labelled accordingly: $C[1-6]$ correspond to quasiordered assemblies, $C[7-11]$ weakly disordered, and $C 12$ fully disordered [35]. Table 1 catalogues the packings used in this work with their respective coordinations. These data were obtained after averaging over five independent realisations.

The major aim of this work is to investigate how packing structure affects the way localised force perturbations are transmitted through sphere packings. In an effort to eliminate other contributing factors that may strongly influence the response properties, a particular geometry is used to study stress transmission. Specifically, to probe the nature of stress transmission inside the packings, a localised, isotropic perturbation was imposed within the central region of the packing by increasing the diameter of the centrally located particles by an amount $\delta d$, in the range $10^{-4} \leq \delta d / d<10^{-2}$. These values for $\delta d$ are directly converted into forces using, $F_{\text {perturb }}=k(\delta d / 2)$. Although this method of perturbation is not standard compared to experimental and previous $2 D$ numerical studies, it provides a convenient method to study the response properties of unconfined packings in the absence of sidewalls and directional external forces such as gravity. Moreover, this method also improves the statistical analysis as discussed below.

For the results presented here to connect with expected theories and experimental works, i.e. to satisfy linearity, it is necessary to gauge the range of the magnitude of the perturbative force that can be applied to the packing. This value was based on the average force per particle, $f_{a v}$, in the static packings prior to the perturbation procedure. These average forces varied over, $10^{-3} k d<f_{\mathrm{av}}<10^{-1} \mathrm{kd}$, from the ordered to most disordered configurations. Thus, the strength of the perturbations was varied from below to above the average force in most cases. After the application of this perturbation, the system was again relaxed at the same initial $\phi=0.742$. This allowed a direct comparison between the initial stress state $\sigma^{\mathrm{i}}$ of the configuration before the perturbation and the stress state $\sigma^{\mathrm{f}}$, of the final configuration after. The contact stresses $\sigma$ were computed for all contacting particles inside a volume $\Omega$ [1], $\sigma_{\alpha \beta}=\frac{1}{\Omega} \sum_{i j} r_{i j}^{\alpha} f_{i j}^{\beta}$, where the $\alpha, \beta$ are the cartesian components of particles $i$ and $j$, separated by $r_{i j}$. The effect of the imposed perturbation was computed as the change in the normal stress between the final and initial states, $\sigma=\sigma^{\mathrm{f}}-\sigma^{\mathrm{i}}$. To reduce statistical uncertainty, for each independent realisation, the stress was averaged over the equivalent faces of the periodic cell, i.e. $\sigma=\left(\sigma_{x x}^{+}+\sigma_{x x}^{-}+\sigma_{y y}^{+}+\sigma_{y y}^{-}+\sigma_{z z}^{+}+\sigma_{z z}^{-}\right) / 6$, where the + and
- represent the normal stresses calculated in the positive and negative direction relative to the position of the perturbation source. See Fig. 1

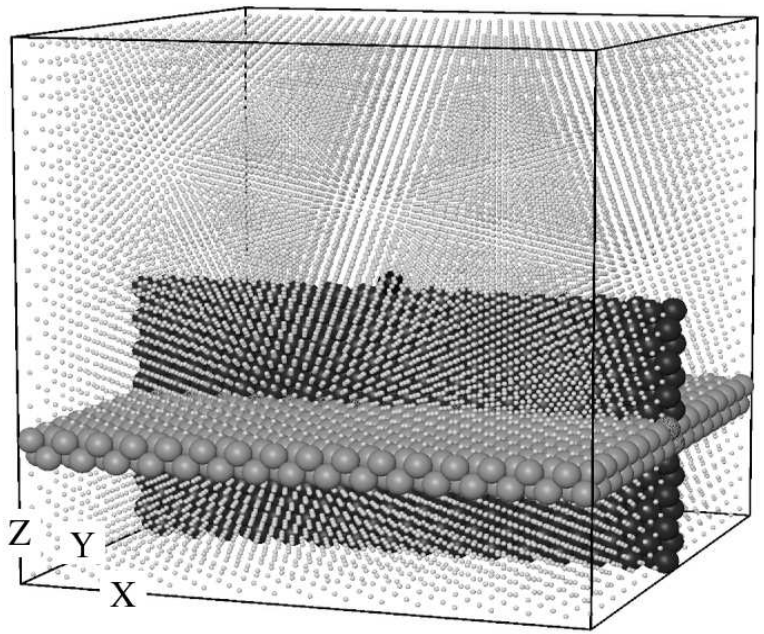

Fig. 1 Schematic of the simulation configuration. The perturbed region is identified by the particle in the middle of the packing. The planes of regular sized spheres represent examples of the visualisation planes for the stress transmission presented in the following figures. All other spheres are drawn at a reduced size for clarity. Axes are labelled although they are all equivalent in this geometry. The simulation cube has side length $L=22.6 \mathrm{~d}$.

Figure 1 presents a schematic of the simulation geometry. The shaded regions represent the planes over which the stresses were computed and visualised in the following figures. The dark gray plane is denoted the direction parallel to the direction of stress transmission, whilst the light gray plane is perpendicular to the transmission direction. The stress response in the plane above the $(+z)$ and below $(-z)$ the point of perturbation are equivalent because, a) the packings are isotropically compressed, b) there is no gravity acting on the system (i.e. directional external forces), and c) because the perturbation itself is isotropic. Likewise, the $+x$ and $-x$ and $+y$ and $-y$ are also all equivalent. Observing the stresses in these planes allows a direct comparison to $2 D$ results. This procedure might appear somewhat artificial, however, there is a close resemblance between this simulation protocol and previous experimental studies. One must simply imagine taking the simulation cube and placing any of its faces on a solid floor and then measuring the pressure profile at this boundary. The benefit of this particular simulation scheme is the ease with which one can gain 'depth' dependent information, where 'depth' is the mean distance from the source of the localised perturbation. Therefore, rather than generating different packings at different heights, one can simply look at different slices within the packing [2]. Moreover, the protocol implemented here could be realised in experiments on colloidal glasses and foams. 


\section{Results}

Figure 2 shows stress response maps for the weakly disordered packing $\mathrm{C} 2$, for one value of the perturbation force. The top panels correspond to different planes perpendicular to the direction of stress transmission (c.f. light gray plane in Fig. 11). The different panels of Fig. 2 show the stress at different 'depths' $h$, with $h=0$ defining the central plane of the packing, coincident with the perturbation source. While the response function gradually decays with distance from the perturbation, in the immediate vicinity of the perturbation (top left panel), the response is localised (single lobe), capturing the finite extent of the imposed perturbation. This dramatically changes character away from the source of the perturbation where the response becomes strongly anisotropic (ringed response). The bottom panel in Fig. 2 shows the plane parallel to the direction of stress transmission (c.f. dark gray plane in Fig. (1) for the same $\mathrm{C} 2$ packing. The response function is directed along characteristic, broadening pathways, leaving the region between these high-stress paths experiencing a relative depletion of stress. This view is similar to the picture put forward in Ref. [30] on stress transmission through ordered arrays of glass beads.
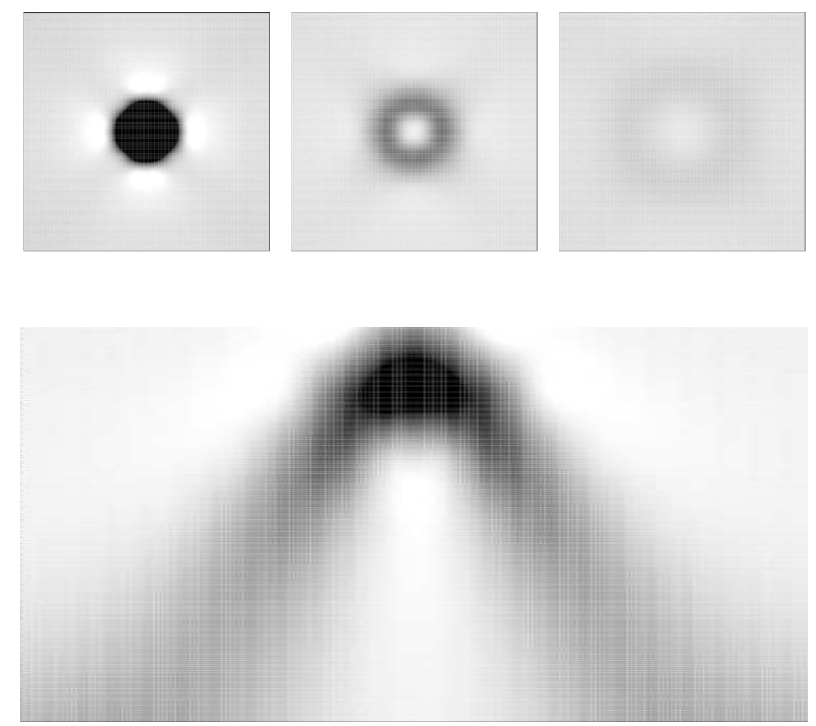

Fig. 2 Stress response inside a weakly disordered packing, $C 2$, due to perturbative force $\delta d=0.0001$. Top panel: Stress maps of approximately $\sim 22.6 d \times 22.6 d$ in size, corresponding to three planes parallel to the light gray plane in Fig. 1 respectively located at $h=2 \sqrt{2}, 3 \sqrt{2}$, and $5 \sqrt{2}$, from left to right. There is a qualitative change in the nature of the response with increasing distance from the perturbation source. The strength of the response also decays with distance from the source. Bottom: Stress map for a central plane corresponding to the dark gray plane in Fig. 1 with dimensions $\sim 22.6 d$ wide along the $x$ axis and extending out $\sim 12 d$ in the $z$ direction away from the perturbation source. Darker shades indicate larger $\sigma$. Intensity scale same in all panels.

To further quantify the nature of the response, and in an effort to compare with equivalent $2 D$ results, the stress profiles shown in Fig. 3 are central cuts taken from (e.g., the top panels in Fig. (2) slices at different $h$ (equivalently, e.g. bottom panel in Fig. (2) inside the $\mathrm{C} 2$ packing for different perturbation magnitudes $\delta d$. This clearly demonstrates that close to the source the response is single-peaked, crossing over to a double-peaked response further away. Not only are the rescaled stresses independent of $\delta d$, but no contacts were broken in the perturbation process, thus confirming the linear response regime. To check the robustness of these results, qualitatively similar data were obtained when $k$ was varied over 5 orders of magnitude, and the degree of overcompression, $\phi-\phi_{\mathrm{fcc}}$, covered 3 orders of magnitude (results not shown here).

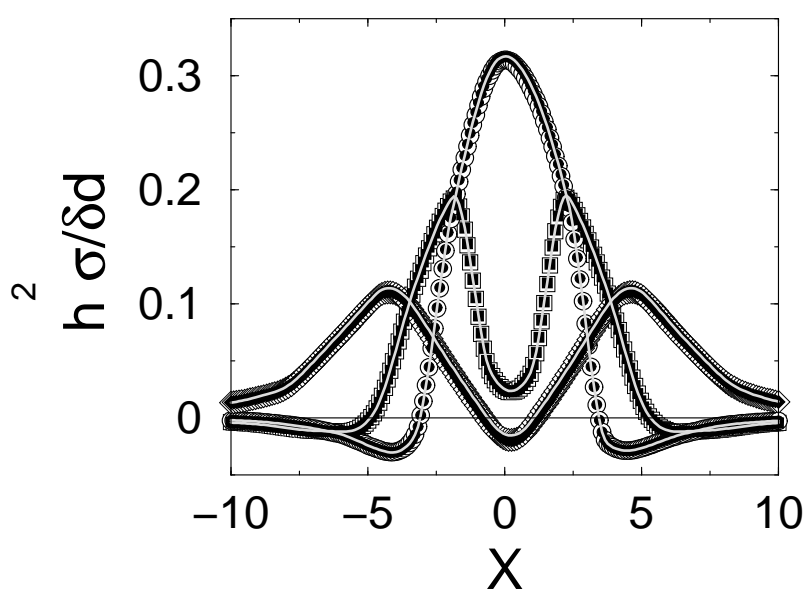

Fig. 3 Stress profiles rescaled by the 'depth', $h$, from perturbation source and the magnitude of the force, $\delta d$, taken from central cuts across the planes shown in the top panels of Fig.2 (packing $C 2$ ). The $x$ axis denotes the distance across the packing face. Each trio of data are for $h=2 \sqrt{2}$ (circles), $3 \sqrt{2}$ (squares), and $5 \sqrt{2}$ (diamonds), for different values of the perturbing force: $\delta d=0.0001$ (open), 0.001 (filled), and 0.01 (grey line).

The results of Figs. 2 and 3 indicate that away from the source of the perturbation, the response functions point toward possible agreement with the hyperbolic wave theory of force propagation [10], which predicts a double-peaked response function with peak widths, $W$, scaling diffusively with depth, $W \sim \sqrt{h}$. To determine which class of theory these results belong to, Fig. 4 shows the depth dependence of $W$ and the peak separations $\Delta r_{\text {peak }}$. From Fig. 4t the characteristic features of the double-peaked response function are seen to vary linearly with depth, $W \sim h$, and therefore do not belong to the hyperbolic class of solutions. This suggests that the response properties of the weakly disordered, frictionless arrays studied here are likely to be described by three dimensional anisotropic elasticity theory.

Although a developed formalism for anisotropic elasticity exists, there are no analytic solutions with which to directly compare these simulation results. Alternatively, an 


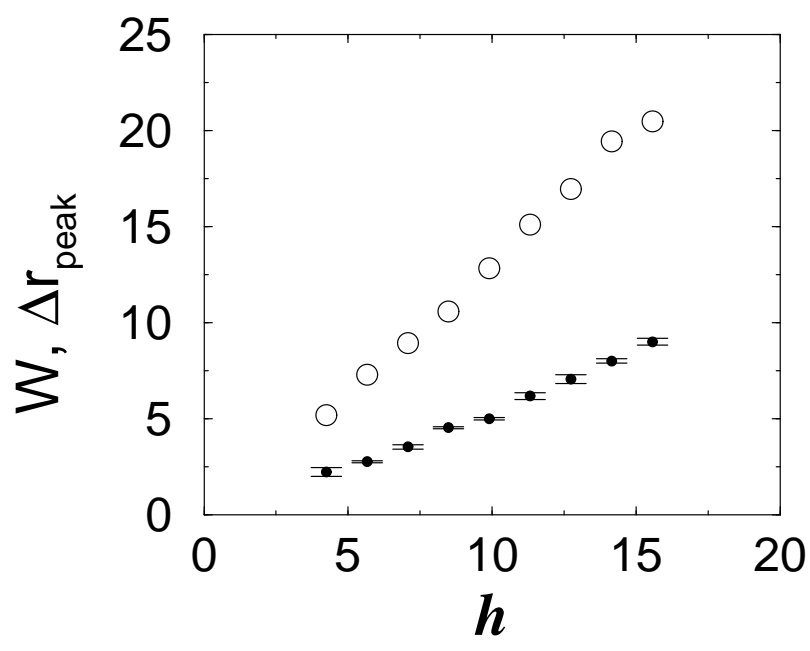

Fig. 4 Stress peak analysis for configuration C2 that exhibits a doublepeak response function. The width $W(\bullet)$, measured at half height of the stress response peak averaged over the two peaks, and the separation between the two peaks, $\Delta r_{\text {peak }}(\mathrm{o})$.

empirical fitting form can be used to describe the data. Motivated by twin-peak, hyperbolic models, Breton et al. [9] introduced the Convection-Diffusion (CD) equation that mimics the response properties of a $2 D$ lattice. The $\mathrm{CD}$ equation is characterised by a propagation, or 'speed', coefficient $c$, that determines the direction of the stress response, and a 'diffusion coefficient' $D$, characterising the diffusive spreading of the two peaks with depth. In its original form, the resulting scalings have the width of the peaks, $W=\sqrt{2 D h}$, consistent with a wave-like hyperbolic picture. This was modified into the Convection-Wave (CW) equation by Geng et al. [15], in an effort to deduce whether their experimental data was better described by the hyperbolic approach or more in line with an elliptic model. To that end, the $\mathrm{CW}$ equation is extended here for $3 D$ systems,

$\sigma(h)=\frac{F}{2(2 \pi)^{3 / 2} \omega^{2} h^{2}}\left(e^{-\Delta_{-}^{2} / 2 \omega^{2} h^{2}}+e^{-\Delta_{+}^{2} / 2 \omega^{2} h^{2}}\right)$.

$F$ is the magnitude of the perturbing force, $r$ is the in-plane radial distance from the central axis of the packing, and $\Delta_{ \pm}=\left(r \pm c^{\prime} h\right)$. Equation2 guarantees a twin peak response with peak widths scaling linearly with depth, $W=\omega h$, where $\omega$ and $c^{\prime}$ depend on the packing structure.

Figure 5 (a) shows the stress response profiles at one depth for a given perturbation, over a range of weak disorder. The double-peak profiles evolve with disorder: the amplitude of the response decreases with increasing disorder, although over this range in disorder, there appears to be little change in the location of the peaks. Equation 2 captures the main features of the profiles - the characteristic double-peak - yet it fails to accurately describe the full response, such as the dip in the profiles either side of the peaks. A representative fit of Eq. 2 (symbols) to the C6 configuration (thick solid line) is shown. A criterion was used in the fitting procedure that attempted to match the peak positions as closely as possible between the simulation data and Eq2. Despite these minor inadequacies, Eq. 2 can be used to characterise the profiles in the weakly disordered regime through the coefficients $c^{\prime}$ and $\omega$, which are obtained as fitting parameters. Over the range of disorder explored in Fig. 5. (a), the values of these fitting parameters are shown in Fig. 5(b). The upper left points correspond to the strongly ordered systems whereas the lower right points correspond to the weakly disordered configurations. These coefficients not only follow the same trend, but are somewhat similar, to those obtained in ordered and weakly-disordered $2 D$ experiments [15].
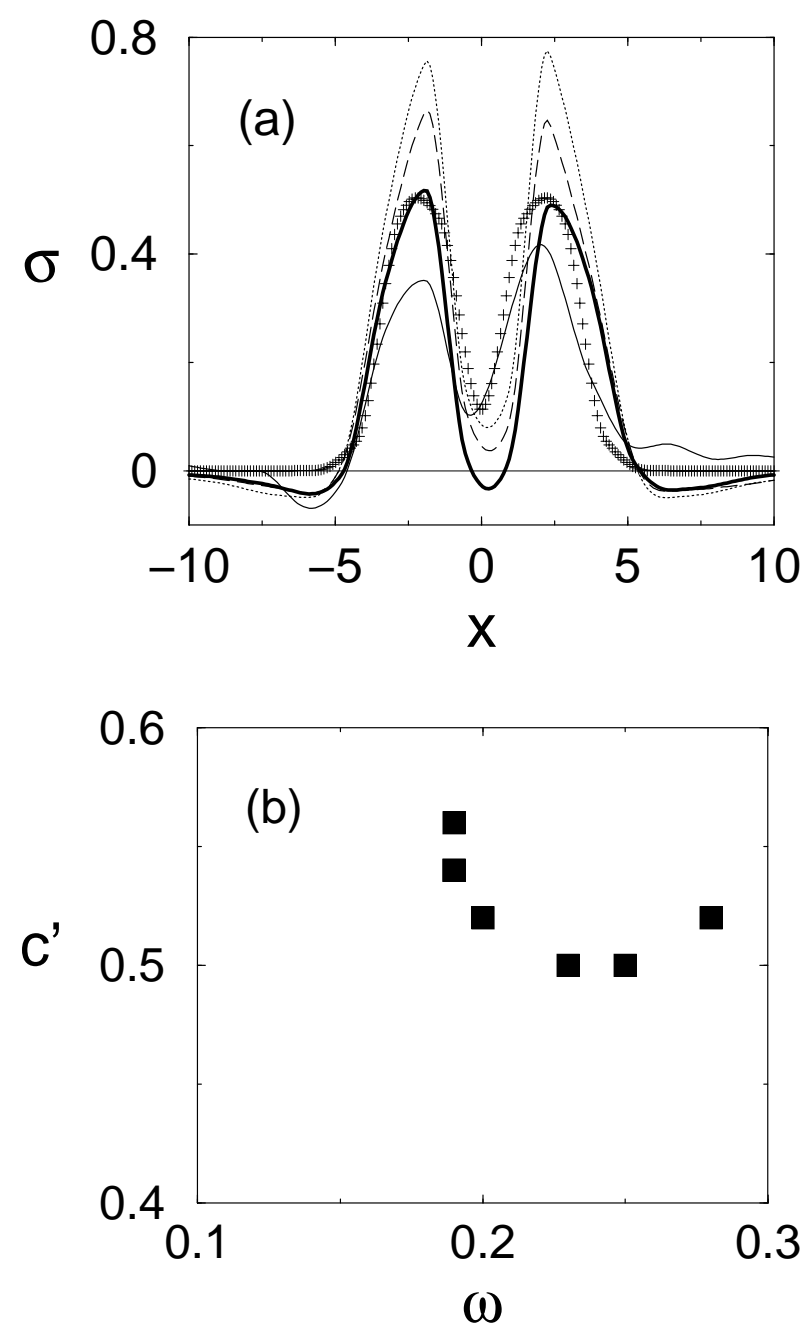

Fig. 5 (a) Scaled stress profiles at $h=3 \sqrt{2}$ for $\delta=0.01$, in the weakly disordered regime. Peak heights decrease with increasing disorder from highest, C2, to lowest, C8. The symbols (+) are a fit of Eq.2 to the data for C6 (thick solid line), with best-fit parameters for $c^{\prime}$ and $\omega$, and is representative of the goodness of the fit for the other configurations. (b) The values of the fitting parameters $c^{\prime}$ and $\omega$ used in Eq. 2 extracted from fitting to the packings $C 2-C 8$. 
When further disorder is introduced, the anisotropic response profiles become less well-defined and there is a crossover to a more isotropic response of the single-peak variety. This is illustrated in the stress maps shown in Fig. 6, for configurations with moderate-large disorder. In an effort to improve averaging over the growing stress fluctuations, both the size of the perturbed region and the averaging volume were varied. It is expected that even for frictionless particles, disorder promotes an isotropic, elastic-like response [19]. For the most disordered system (C12), whose structure resembles that of an overcompressed random closed packed state, a single-peaked response can be clearly resolved. This is better illustrated in the profile shown in Fig.7 7 A comparison to the Boussinesq theory of Eq. 11 shows reasonable agreement [3]. Further promoting the idea that isotropic random packings are more likely to exhibit isotropic elastic-like stress behaviour.
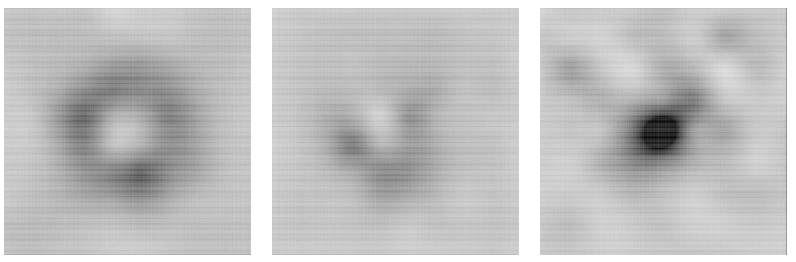

Fig. 6 Stress response at $h=3 \sqrt{2}$ for $\delta d=0.001$, inside packings with increasing disorder from left to right: $C 8, C 11$, and $C 12$. Size of slice $\sim 20 d \times 20 d$. Shading is the same in all panels.

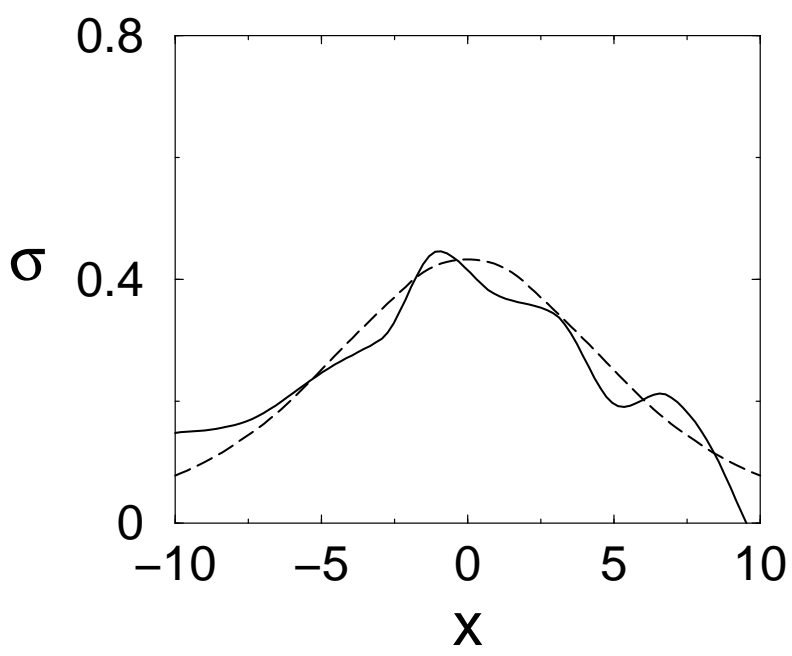

Fig. 7 Comparison between the Boussinesq theory, Eq. 1 (dashed line) and the stress response for the most disordered configuration $\mathrm{C} 12$, which resembles an overcompressed random close packing. At $h=3 \sqrt{2}$ inside the packing for $\delta d=0.001$.

\section{Discussion}

The procedure described here presents only one of a number of avenues through which the mechanical response of a material may be investigated. Traditionally, shear tests have been used to determine constitutive parameters through which material, or elastic, constants can be obtained from linear stress-strain relations. Moreover, for the explicit study of response properties of the type studied here, the elastic constants must be initially determined which can then be input into finite element analyses routines [4]. However, in terms of computational time such procedures are just as costly, if not more so, as the perturbation protocol implemented here.

To reiterate, the goal here was to make a connection with experimental studies on similar systems. The results presented here are the first in a series of investigations on the mechanical response in particulate media. In this initial study it has been shown that the force response method provides a quantitative description of the elastic properties of particle packings consistent with previous studies. To that end, the protocol implemented here has been verified as a suitable procedure through which we can investigate mechanical response problems. Even though the particular geometry used is not conventional from an experimental standpoint it does offer a method to determine the influence of different parameters on the mechanical behaviour of sphere packings. The focus here was structure and is likely relevant not only to compressed elastic sphere-packings, but also foam dispersions.

\section{Conclusions}

For the first time, a systematic study of the nature of stress transmission in response to localised force perturbations has been obtained inside three dimensional packings of frictionless spheres using discrete element simulations, in a particular geometry that avoids the influence of walls. The manner in which stresses are transmitted crucially depends on the distance from the source of the perturbation and the structure of the packing. For small deviations from the fcc structure, the response in the vicinity of the perturbation source retains the localised form of the perturbation, and appears as a single-peak response function. This crosses over to an anisotropic response function of the double-peak variety further from the source. This double-peak feature is suitably captured by the modified Convection-Wave equation; peak widths scaling linearly with distance, rather than the diffusive form of this equation [9]. These results are consistent with a generalisation from $2 D$ to $3 D$ anisotropic elasticity theories [32]. Further disorder deforms the double-peak features associated with the anisotropic response function until there is a crossover to a single-peak response for randomly 
packed spheres consistent with an isotropic elastic description. Moreover, this crossover is qualitatively matched by the Boussinesq equation, Eq. 1, which again indicates that the procedure implemented here probes mechanical properties that is simple and quite accurate [41]. This will be particularly useful in future studies on random packings with different coordination numbers with and without friction.

It is known experimentally the stress response of a disordered, frictional granular packing qualitatively agrees with isotropic elasticity theory [33]. Simulations of two dimensional systems also point towards agreement with isotropic elastic models [19]. Studies of three dimensional frictional ordered and disordered packings and gravity packings remains work in progress.

Acknowledgements I thank P. Claudin and J. Socolar for fruitful discussions, and one of the referees for their detailed and supportive critique. I gratefully acknowledge the support of an ORDA Faculty Seed Grant at SIU and the National Science Foundation under Grant No. CBET-0828359.

\section{References}

1. For the weakly disordered systems, the results are insensitive to the choice of $\Omega$, provided it is larger than the interparticle spacing. In that case $\Omega=\sqrt{2}$. For more disordered configurations, $\Omega=$ $\frac{3}{2} \sqrt{2}$.

2. The results presented here are qualitatively similar to packings in a gravitational field resting on a solid boundary.

3. A fitting of the data to the response expected within an infinite elastic medium was also attempted, with the Poisson ratio of the material now entering as a free parameter [26; 40]. This excercise did not shed any further light on the analysis.

4. Atman APF, Brunet P, Geng J, Reydellet G, Claudin P, Behringer RP, Clement E (2005) From the stress response function (back) to the sand pile "dip". Eur Phys J E 17:93

5. Atman APF, Brunet P, Geng J, Reydellet G, Claudin P, Behringer RP, Clement E (2005) Sensitivity of the stress response function to packing preparation. J Phys:Condens Matter 17:S2391

6. Blumenfeld R (2004) Stresses in isostatic granular systems and emergence of force chains. Phys Rev Lett 93:108,301

7. Bonamy D, Bernard-Bernardet S, Daviaud F, Laurent L (2003) Microdisplacements induced by a local perturbation inside a granular packing. Phys Rev E 68:042,301

8. Bouchaud JP, Claudin P, Levine D, Otto M (2001) Force chain splitting in granular materials: A mechanism for large-scale pseudo-plastic behaviour. Eur Phys J E 4:451

9. Breton L, Claudin P, Clement E, Zucker JD (2002) Stress response function of a two-dimensional ordered packing of frictional beads. Europhys Lett 60:813

10. Claudin P, Bouchaud JP, Cates ME, Wittmer JP (1998) Models of stress fluctuations in granular media. Phys Rev E 57:4441

11. Coppersmith SN, h Liu C, Majumdar S, Narayan O, Witten TA (1996) Model for force fluctuations in bead packs. Phys Rev E 53:4673

12. Eloy C, Clement E (1997) Stochastic aspects of the force network in a regular granular piling. $J$ Phys I France 7:1541

13. Gay C, da Silveira R (2004) Anisotropic elastic theory of preloaded granular media. Europhys Lett 68:51
14. Geng J, Howell D, Longhi E, Behringer RP, Reydellet G, Vanel L, Clement E, Luding S (2001) Footprints in sand: The response of a granular material to local perturbations. Phys Rev Lett 87:035,506

15. Geng J, Reydellet G, Clement E, Behringer RP (2003) Green's function measurements of force transmission in $2 \mathrm{~d}$ granular materials. Physica D 182:274

16. Gland N, Wang P, Makse HA (2006) Numerical study of the stress response of two-dimensional dense granular packings. Eur Phys $J$ E 20:179

17. Goldenberg C, Goldhirsch I (2002) Force chains, microelasticity, and macroelasticity. Phys Rev Lett 89:084,302

18. Goldenberg C, Goldhirsch I (2004) Small and large scale granular statics. Gran Matt 9:245

19. Goldenberg C, Goldhirsch I (2005) Friction enhances elasticity in granular solids. Nature 435:188

20. Goldenberg C, Goldhirsch I (2008) Effects of friction and disorder on the quasistatic response of granular solids to a localized force. Phys Rev E 77:041,303

21. Goldhirsch I, Goldenberg C (2002) On the microscopic foundations of elasticity. Eur Phys $J$ E 9:245

22. Head DA, Tkachenko AV, Witten TA (2001) Robust propagation direction of stresses in a minimal granular packing. Eur Phys $J$ E 6:99

23. Jaeger HM, Nagel SR, Behringer RP (1996) Granular solids, liquids, and gases. Rev Mod Phys 68:1259

24. Johnson KL (1987) Contact Mechanics. Cambridge University Press, Cambridge

25. Kenkre VM, Scott JE, Pease EA, Hurd AJ (1998) Nonlocal approach to the analysis of the stress distribution in granular systems. I. Theoretical framework. Phys Rev E 57:5841

26. Landau LD, Lifshitz EM (1986) Theory of Elasticity, Course of Theoretical Physics, vol 7, 3rd edn. Elsevier, Oxford

27. Leonforte F, Tanguy A, Wittmer JP, Barrat JL (2004) Continuum limit of amorphous elastic bodies II: Linear response to a point source force. Phys Rev B 70:014,203

28. Leonforte F, Boissiere R, Tanguy A, Wittmer JP, Barrat JL (2005) Continuum limit of amorphous elastic bodies. III. Threedimensional systems. Phys Rev B 72:224,206

29. Moukarzel CF, Pacheco-Martinaez H, Ruiz-Suarez JC, Vidales AM (2004) Static response in disk packings. Gran Matt 6:61

30. Mueggenburg NW, Jaeger HM, Nagel SR (2002) Stress transmission through three-dimensional ordered granular arrays. Phys Rev E 66:031,304

31. Ostojic S, Panja D (2005) Response of a hexagonal granular packing under a localized external force. Europhys Lett 71:70

32. Otto M, Bouchaud JP, Claudin P, Socolar JES (2003) Anisotropy in granular media: Classical elasticity and directed-force chain network. Phys Rev E 67:031,302

33. Reydellet G, Clement E (2001) Green's function probe of a static granular piling. Phys Rev Lett 86:3308

34. Silbert LE (2005) Structural dependency on force propagation through particle packings. In: RGarca-Rojo, Herrmann H, McNamara S (eds) Powders \& Grains 2005, A.A.Balkema, Rotterdam, vol $1, \mathrm{p} 99$

35. Silbert LE (2006) Force heterogeneities in particle assemblies: From order to disorder. Phys Rev E 74:041,304

36. Silva MD, Rajchenbach J (2000) Stress transmission through a model system of cohesionless elastic grains. Nature 406:708

37. Socolar JES, Schaeffer DG, Claudin P (2002) Directed force chain networks and stress response in static granular materials. Eur Phys $J E$ 7:353

38. Spannuth MJ, Mueggenburg NW, Jaeger HM, Nagel SR (2004) Stress transmission through three-dimensional granular crystals with stacking faults. Gran Matt 6:215

39. Tanguy A, Wittmer JP, Leonforte F, Barrat JL (2002) Continuum limit of amorphous elastic bodies: A finite-size study of low fre- 
quency harmonic vibrations. Phys Rev B 66:174,205

40. Timoshenko SP, Goodier JN (1970) Theory of Elasticity, 3rd edn. McGraw-Hill

41. Tsamados M, Tanguy A, Goldenberg C, Barrat JL (2009) Local elasticity map and plasticity in a model Lennard-jones glass. Phys Rev E 80:026,112

42. Walsh SDC, Tordesillas A (2004) The stress response of a semiinfinite micropolar granular material subject to a concentrated force normal to the boundary. Gran Matt 6:27

43. Wittmer JP, Tanguy A, Barrat JL, Lewis L (2002) Vibrations of amorphous nanometric structures: When does continuum theory apply? Europhys Lett 57:423

44. h Yi C, Liu Y, d Miao T, s Mu Q, 1 Qi Y (2007) Force transmission in three-dimensional hexagonal-close-packed granular arrays with point defects submitted to a point load. Gran Matt 9:1 